\title{
Tecnura
}

INVESTIGACIÓN

\section{Implementación del método de escasez en la determinación de la huella hídrica en la zona costera de San Blas, México}

\section{Implementation of the shortage method \\ to determine the water footprint in the coastal area of San Blas, México}

\author{
Sara Edith Bueno Pérez ${ }^{(}$, Susana Marceleño Flores $^{2}{ }^{\circledR}$, Oyolsi Nájera González ${ }^{(}$ \\ y Rebeca de Haro Mota ${ }^{4}$
}

Fecha de recepción: 17 de mayo de 2019

Fecha de aceptación: 23 de agosto de 2019

Como citar: Bueno, S.E., Marceleño, S., Nájera O. y De Haro, R. (2019). Implementación del método de escasez en la determinación de la huella hídrica en la zona costera de San Blas, México. Tecnura, 23(62), 45-54. https:// doi.org/10.14483/22487638.15796

\section{Resumen}

Contexto: El agua es esencial para la vida y la conservación de su calidad es vital. La cantidad de agua dulce existente en la Tierra es limitada, y su calidad está sometida a una presión constante. Esta problemática hace necesario generar herramientas que permitan medir los impactos potenciales que causan las actividades humanas sobre el recurso hídrico. Por ello, esta investigación determina la huella hídrica en la zona costera de San Blas, México.

Método: Este trabajo estima la huella de agua en la zona costera del municipio de San Blas, Nayarit (México), a partir del método de escasez de agua, que tiene lugar cuando la demanda supera el suministro de agua dulce en un área determinada. Este método considera el índice de estrés hídrico en su cálculo.

Resultados: La huella de agua para cuantificar el volumen total del líquido utilizado por los habitantes resulta inferior al promedio nacional. De esta manera, la zona de estudio no presenta estrés hídrico, ya que la extracción de agua no ha superado su disponibilidad.

Conclusiones: La huella hídrica por el método de escasez puede considerarse como un indicador de la proporción de extracciones anuales y disponibilidad de agua; permite realizar comparaciones de la presión a la que está sometido el recurso hídrico entre zonas, regiones y países. Además, puede contribuir a la planificación de la distribución y gestión del agua en uso agrícola y urbano, en regiones como este estudio, donde el área de uso agrícola supera el 35 \%. La estimación de la huella hídrica presentada en este trabajo considera la relación entre la extracción de agua dulce para uso humano y su disponibilidad total de agua en una región dada (WSI), el volumen distribuido y el porcentaje de cobertura de agua potable del área en estudio.

Palabras clave: escasez, estrés, huella hídrica, método, sustentabilidad.

1 Estudiante de la Licenciatura en Biología, Unidad Académica de Agricultura. Universidad Autónoma de Nayarit. Tepic, México. Contacto: edith_711@uan.edu.mx

2 Profesor investigador, Cuerpo Académico Recursos Naturales. Universidad Autónoma de Nayarit. Tepic, México. Contacto: smlmarcel@uan. edu.mx

3 Profesor investigador, Cuerpo Académico Recursos Naturales. Universidad Autónoma de Nayarit. Tepic, México. Contacto: oyolsi@uan.edu.mx

4 Profesor investigador, Cuerpo Académico Recursos Naturales. Universidad Autónoma de Nayarit. Tepic, México. Contacto: deharo@uan. edu.mx 


\section{Abstract}

Context: Water is essential for life and conservation of water quality is vital. The amount of fresh water on earth is limited and its quality is subject to constant pressure. This problem makes it necessary to present tools that allow quantifying the possible impacts generated by human activities in this resource. Therefore, this research determines the water footprint in the coastal area of San Blas, Mexico.

Method: This work estimates the water footprint in the coastal area of he municipality of San Blas, Nayarit, Mexico, according to the method of water scarcity that occurs when demand exceeds the supply of fresh water in a given area. This method considers the water stress index in its calculation.

Results: The result shows that the water footprint to quantify the total volume of water used by the inhabitants is less than the national average. In this way, the study area does not present water stress, since the extraction of water has not exceeded its availability.

Conclusions: The water footprint by the scarcity method can be considered as an indicator of the proportion of annual withdrawals and water availability; It allows comparisons of the pressure to which the water resource is subjected between zones, regions and countries. In addition, it can contribute to the planning of the distribution and management of water in agricultural and urban use, in regions such as this study, where the area of agricultural use exceeds $35 \%$. The estimation of the water footprint presented in this work considers the relationship between the extraction of fresh water for human use and its total water availability in a given region (WSI), the volume distributed and the percentage of drinking water coverage of the area in study.

Keywords: shortage, stress, water footprint, method, sustainability.

\section{INTRODUCCIÓN}

La Organización de las Naciones Unidas para la Alimentación y la Agricultura (FAO) advierte que el agua es uno de los mayores desafíos del siglo $X X I$, ya que es esencial para la producción agrícola, y además constituye el elemento vital de los ecosistemas. De continuar con los hábitos actuales, se estima que la demanda mundial de agua podría aumentar un 50 \% para 2030 (FAO, 2018). Por su parte el Reporte de Riesgos Globales (World Economic Forum, 2016) advierte que la crisis del agua se encuentra dentro de los diez riesgos más relevantes para la humanidad con respecto al impacto que podría generar y la probabilidad de ocurrencia, y constituye el mayor riesgo vislumbrado en la próxima década.

"Los eventos climáticos extremos ocasionan grandes retos para la sostenibilidad social, económica y medioambiental" (González, Carvajal y Loiza, 2016, p. 101). En este sentido, los efectos de la crisis del agua se relacionan con los tres ejes del desarrollo sustentable. En el sector económico, la crisis del agua representa un riesgo para las empresas que requieren grandes cantidades de agua dentro de sus procesos industriales y comerciales. En cuestiones sociales, genera disrupción social, problemas de salud, migraciones forzadas e, incluso, conflictos entre comunidades, debido en parte a la ausencia de una concepción sistémica en la planificación de la ciudad y el territorio (De Quevedo, Asprilla y González, 2017, p. 138). Además, en el sector ambiental se han producido diversos efectos como la pérdida de biodiversidad con repercusiones en los ecosistemas. Y es aquí, donde "México por su lado, padece una serie de problemas ambientales que comprometen la sostenibilidad de su desarrollo" (González, Retamoza, Álbores y Guerrero, 2016, p. 92).

En función de lo anterior, el agua como elemento esencial para la vida y la conservación de su calidad es vital. Asimismo, la cantidad de agua dulce existente en el planeta es restringida, y su calidad permanece con una presión constante. 
Esta problemática hace necesario presentar herramientas que permitan medir los impactos potenciales que causan las actividades humanas sobre este recurso. La crisis hídrica que actualmente afronta el planeta encuentra solución en el ámbito académico a través del desarrollo de indicadores que facilitan evaluar el uso y la disposición del agua. Asimismo, muchos países han desarrollado legislación ambiental para limitar los vertidos que han deteriorado paulatinamente el recurso hídrico (Suarez, García y Vaca, 2012, p. 186).

En el tema del agua existen diferentes indicadores e índices: resources to population index (Falkenmark, 1989), indicator of relative water scarcity (Seckler, Molden y Barker, 1999), water scarcity index (Gleick, 1996), social water stress index (Ohlsson, 2000), water poverty index (Sullivan, 2002). Sin embargo, existe una propuesta como herramienta de evaluación de la sostenibilidad de los recursos hídricos, que se emplea para cuantificar el volumen total de agua utilizada en todas las actividades de los habitantes de una determinada región, esta es la huella de agua o huella hídrica.

El término huella nació en la década de 1990 bajo el concepto de huella ecológica, que es previo a la huella de agua, y fue introducido por William Rees y Mathis Wackernagel (Rees, 1992, 1996). La huella ecológica de una población describe el área de tierra productiva y ecosistemas acuáticos necesarios para producir los recursos empleados por esta; además, considera el área necesaria para eliminar sus residuos, pero no el lugar geográfico en que dichas áreas se encuentren, solo toma un determinado nivel de vida. De esta forma, la huella ecológica se refiere a la cantidad de superficie de terreno necesaria para que una población o comunidad pueda desarrollarse de forma sostenible.

A su vez, J.A. Allan (1998) usa por primera vez el concepto agua virtual al buscar solución a los problemas de escasez de este recurso en Medio Oriente. En su trabajo observa que los productos elaborados requerían de una gran cantidad de agua en su proceso de elaboración, además de la que se encuentra en ellos como producto final.
El concepto agua virtual lo retoman Arjen Y. Hoekstra y Ashok Chapagain (2002) aplicado a un concepto nuevo: la huella de agua (Hoekstra, 2003; Hoekstra y Chapagain, 2004). La metodología propuesta por Hoekstra, publicada por la iniciativa Water Footprint Network (WFN), se creó para lograr un indicador que relacionara el agua con el uso y consumo de las personas. La huella de agua puede ser externa, si se considera la cantidad del líquido necesario para desarrollar los productos consumidos en un país, cuando estos han sido elaborados en el exterior; o puede ser interna, si se tiene en cuenta solo el líquido extraído de los recursos nacionales del país que se observa. También, muestra los efectos sobre la disponibilidad y la escasez de agua.

En la actualidad, existen diversas metodologías para reportar la huella de agua: a) por volumen, b) por impactos y c) por escasez. En América Latina, para evaluar la huella de agua, se ha usado principalmente la metodología de escasez en agua dulce, elaborada por Pfister, Koehler y Hellweg (2009), ajustando el factor del water stress index (WSI) para cada zona hidrográfica (Martínez et al., 2016). En la tabla 1 se presentan algunos métodos por escasez y sus características.

El método de escasez (Pfister, Koehler y HeIlweg, 2009) calcula qué tan estresado está el recurso en determinada zona, tomando en cuenta la cantidad de agua superficial y subterránea que se utiliza para satisfacer las necesidades de la población. La escasez de agua puede describirse en términos generales como una escasez en la disponibilidad de agua dulce renovable en relación con la demanda (Taylor, 2009). Se presenta cuando la demanda supera el abastecimiento de agua dulce en un área determinada (FAO, 2018). La cuantificación formal de la escasez de agua comenzó a principios de la década de 1980 con el desarrollo del índice de estrés hídrico (WSI) que vincula explícitamente la seguridad alimentaria con la disponibilidad de agua dulce (Falkenmark 1986, 1989). Este índice se ha convertido, desde entonces, en la medida de escasez de agua más ampliamente aplicada (Damkjaer y Taylor, 2017). 
Tabla 1. Métodos para el cálculo de la escasez hídrica

\begin{tabular}{|c|c|}
\hline Método & Descripción \\
\hline $\begin{array}{l}\text { Consumo equivalente-Índice } \\
\text { de estrés hídrico (WSI), Pfister } \\
\text { et. al. (2009). }\end{array}$ & $\begin{array}{l}\text { EI WSI muestra la relación entre la extracción de agua dulce para usos humanos y la disponibi- } \\
\text { lidad total de agua en cierta región. } \\
\text { El indicador puede tener un valor igual al del consumo de agua. WSI se ubica entre } 0,01 \text { (el } \\
\text { cual representa efecto marginal) y } 1 \text {, para lograr este rango utiliza una ecuación logarítmica. }\end{array}$ \\
\hline $\begin{array}{l}\text { Consumo equivalente-AWA- } \\
\text { RE, Boulay et al. (2015). }\end{array}$ & $\begin{array}{l}\text { AWARE es un factor de caracterización basado en la relación del consumo de agua dulce para } \\
\text { usos humanos y el caudal ambiental con la disponibilidad de agua en área específica. } \\
\text { Este indicador podría alcanzar } 100 \text { veces el agua consumida. AWARE tiene un rango entre } 0,1 \\
\text { y } 100 \text {. Si el factor de caracterización de una región es } 10 \text {, indica que esa región tiene } 10 \text { veces } \\
\text { menos agua remanente disponible que el promedio mundial. }\end{array}$ \\
\hline $\begin{array}{l}\text { Escasez de huella azul, Hoe- } \\
\text { kstra, Chapagain, Aldaya y } \\
\text { Mekonnen (2011). }\end{array}$ & $\begin{array}{l}\text { Representa la proporción de agua consumida respecto a la disponibilidad de agua azul. En este } \\
\text { caso la disponibilidad es el agua total disponible en la cuenca menos el caudal ambiental. } \\
\text { Para algunas cuencas, este indicador de escasez de huella azul alcanza } 675 \text { veces el agua con- } \\
\text { sumida (según datos reportados por la WFN). }\end{array}$ \\
\hline $\begin{array}{l}\text { Método suizo de escasez } \\
\text { ecológica, Frischknecht et al. } \\
\text { (2006). }\end{array}$ & $\begin{array}{l}\text { Este método muestra la distancia que falta por recorrer para alcanzar los objetivos políticos, } \\
\text { asociando }\end{array}$ \\
\hline $\begin{array}{l}\text { Consumo de agua que afecta } \\
\text { ecosistemas de río, Hanafiah } \\
\text { et al. (2011). }\end{array}$ & $\begin{array}{l}\text { Este indicador se basa en un modelo global que considera volúmenes de los ríos, tiempo pro- } \\
\text { medio de residencia, descarga promedio y especies de peces en varias cuencas del mundo. }\end{array}$ \\
\hline $\begin{array}{l}\text { Consumo de agua subterránea } \\
\text { que afecta plantas, Van Zelm } \\
\text { et al. } 2010\end{array}$ & $\begin{array}{l}\text { Es un método para calcular los factores relacionados con los efectos de la extracción de agua } \\
\text { subterránea en la riqueza de especies de plantas terrestres. }\end{array}$ \\
\hline $\begin{array}{l}\text { Enfermedades infecciosas por } \\
\text { escasez de agua doméstica, } \\
\text { Motoshita, Itsubo e Inaba } \\
(2010) \text {. }\end{array}$ & $\begin{array}{l}\text { En este método se modela la evaluación de daños a la salud por enfermedades infecciosas } \\
\text { causadas por la escasez de agua doméstica. Usa indicadores socioeconómicos y de desarrollo. }\end{array}$ \\
\hline
\end{tabular}

Fuente: adaptada de Martínez et al. (2016).

El método de escasez requiere del cálculo del índice de estrés hídrico (WSI), del grado de presión hídrica (WTA) y de este último en base a la precipitación anual (WTA*). Cuando la huella de agua está orientada al estrés hídrico, se integra el volumen total del líquido utilizado y los impactos ambientales provocados en la elaboración de un producto. El estrés hídrico se define comúnmente por la relación entre las extracciones anuales totales de agua dulce y la disponibilidad hidrológica.

El grado de estrés es un indicador expresado en porcentaje, de la presión a la que se encuentra sometido el recurso hídrico en un país, cuenca o región. Para Vorosmarty, Green, Salisbury y Lammers (2000), y Alcamo, Henrichs y Rosch (2000), el estrés hídrico moderado y severo ocurre por encima de un umbral de $20 \%$ y $40 \%$, respectivamente. Otra fuente registra como presión fuerte, si el porcentaje es mayor a 40 \% (Conagua, 2017).
En este con contexto, el presente trabajo tiene como objetivo evaluar la huella de agua de la zona costera de San Blas, Nayarit (México), mediante el método de escasez de agua. La zona de estudio se ubica en San Blas, municipio y puerto del estado mexicano de Nayarit. La zona costera de San Blas está inmersa en la costa del Pacífico mexicano, se localiza en la región norte del estado de Nayarit; el tipo de clima que presenta es cálido-subhúmedo con precipitación promedio anual de $1.316,3 \mathrm{~mm}$, la temperatura promedio anual es de $25,6{ }^{\circ} \mathrm{C}$. El área en estudio se caracteriza por tierras de cultivo (37,5\%), áreas de manglar $(19,67 \%)$ y vegetación secundaria $(13,55 \%)$. En menor proporción, se presentan áreas de pastizal, selvas y bosques. Los asentamientos humanos y la infraestructura acuícola representan $2,45 \%$ y $4,38 \%$, respectivamente, como se muestra en la figura 1. 

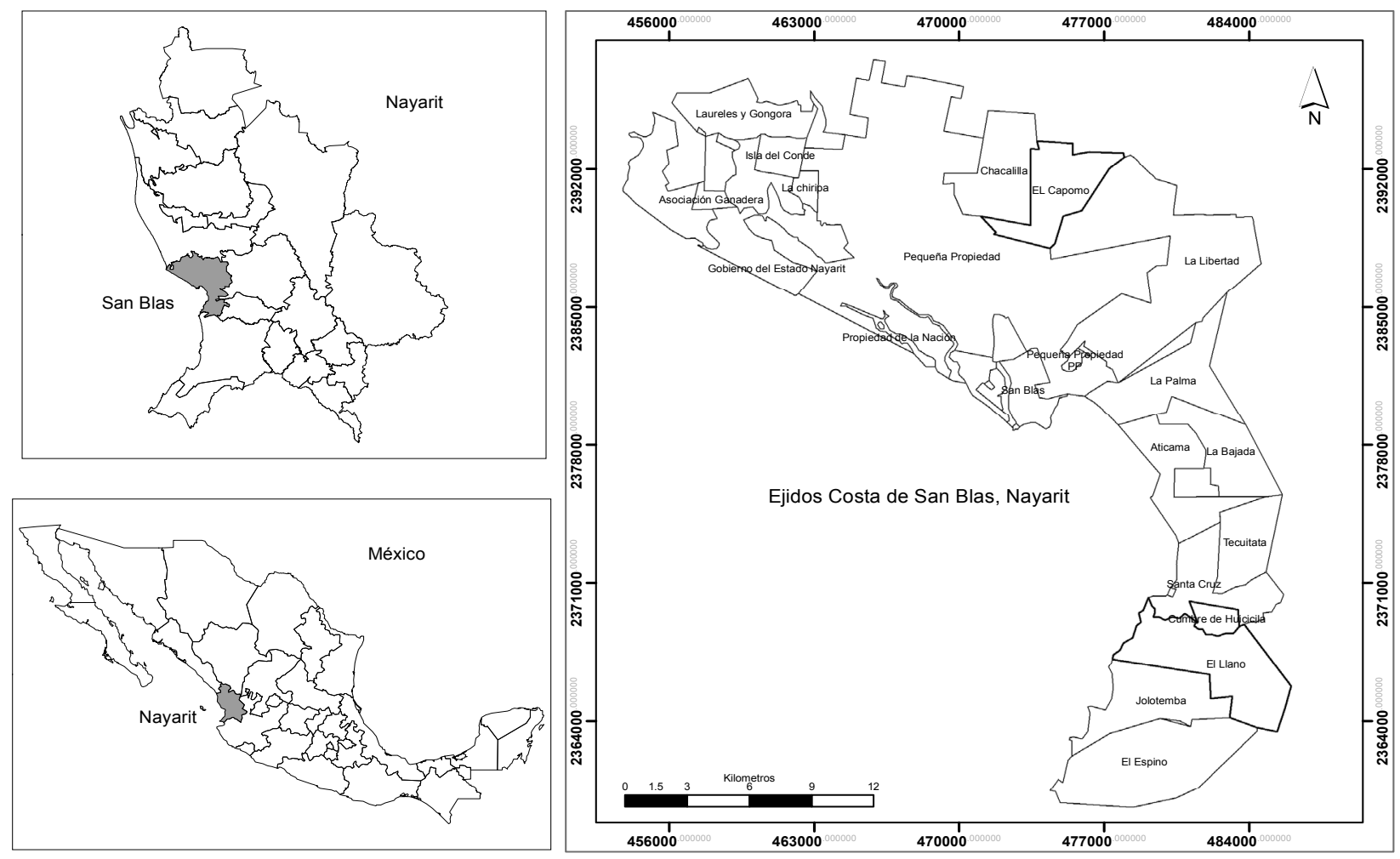

Figura 1. Área de estudio de San Blas, México

Fuente: elaboración propia.

En la zona costera de San Blas habitan 14.195 personas distribuidas en 22 localidades. En materia social se advierten algunos rezagos. De acuerdo con el Consejo Nacional de Población (Conapo), en 2010, el grado de marginación de las localidades oscilaba entre alto, medio y bajo.

La estimación del índice de marginación se hace a partir de la información de los Censos y Conteos de Población y Vivienda elaborados por el INEGI. Este indicador mide las disparidades territoriales y las desigualdades sociales que existen a nivel estatal y municipal en el país, creado con el interés particular de ser una medida que dé cuenta de las carencias que padece la población, como: porcentaje de la población que carece de educación, servicios en la vivienda, percepción bajos ingresos y la residencia en las pequeñas localidades. Por otra parte, en la zona de estudio, en 2010, el rezago social se encontraba entre bajo y muy bajo.
El índice de rezago social es una medida ponderada que resume cuatro indicadores de carencias sociales (educación, salud, servicios básicos y espacios en la vivienda) en un solo índice que tiene como finalidad ordenar a las unidades de observación según sus carencias sociales.

En el ámbito económico, en la zona costera de San Blas se observa una dependencia económica de 135; es decir que cada 135 personas inactivas (sin empleo formal) son sostenidas por cada 100 personas activas (con empleo formal). La tasa bruta de actividad económica es de 42 , es decir que el $42 \%$ de la población total está ocupada en alguna actividad productiva. La tasa bruta de actividad económica es mayor en hombres que en mujeres (59 \% y $25 \%$, respectivamente). Asimismo, en el aspecto económico, según el Directorio Estadístico Nacional de Unidades Económicas (DENUE), en la zona costera de San Blas hay 1887 
unidades económicas; mayormente son actividades terciarias de diversos giros, principalmente los de distribución de bienes y servicios relacionados con la recreación. De las actividades secundarias, la que se registra es la de transformación de bienes, principalmente en la industria manufacturera. Por otra parte, la explotación de recursos naturales es la característica que representa a la actividad primaria, por lo que dichos análisis se realizaron mediante un software comercial que facilita el diseño de las intersecciones, el acceso de vehículos y radios de giro.

\section{METODOLOGÍA}

La metodología aplicada para el cálculo de la huella de agua fue la propuesta por Pfister, Koehler y Hellweg (2009), la cual indica qué tan estresado está el recurso agua en determinada zona. La ecuación (1) describe la fórmula general de la huella de agua aplicando dicha metodología:

$\mathrm{HA}=(\mathrm{WSI})$ (volumen distribuido) * (\% de cobertura de agua potable)

Para obtener el valor del índice de estrés hídrico (WSI), fue necesario estimar el grado de presión hídrica (WTA) de la zona y el grado de presión hídrica en base a la precipitación anual (WTA*), y obtener algunos datos como la cantidad de extracción de aguas tanto superficiales como subterráneas, además de la disponibilidad de agua y la población total de área de estudio.

El cálculo del grado presión hídrica (WTA) está representado por la ecuación (2):

$$
W T A_{i}=\frac{\sum_{J} W U_{i j}}{W A_{i}}
$$

Donde:

$i=$ cuenca.

$j=$ los diferentes usuarios (agricultura, industria y habitantes).

$W A_{i}=$ disponibilidad del agua.

$W U_{i j}=$ extracciones realizadas por los usuarios.
La ecuación (2) expresa la cuantificación del grado de estrés hídrico (WTA) de una cuenca $i$ donde diferentes usuarios $j$ (industria, agricultura y habitantes) extraen agua, se realiza a partir de la disponibilidad anual de agua $\left(W A_{i}\right)$ y de las extracciones realizadas por los diferentes usuarios $\left(W U_{i j}\right)$, respectivamente para cada cuenca $i$.

Para calcular algunas de las variables, se requirió el número de habitantes de la zona costera de San Blas, que de acuerdo con el censo realizado por el INEGI en 2010, el número de habitantes fue de 14.195 personas. En este sentido, para estimar el volumen de extracción de agua, tanto de mantos acuíferos superficiales como subterráneos para la zona de estudio, se consideró el volumen de extracción de agua superficial y subterránea anual del estado. Se hizo una corrección mediante una distribución per cápita y considerando la población de la zona costera de San Blas.

Por otro lado, para obtener el valor de disponibilidad de agua, se consultó en el Diario Oficial de la Federación, el Acuerdo (Publicado el 12 de julio de 2013) por el que se actualiza la disponibilidad media anual de las aguas nacionales superficiales. Según este documento, se actualiza la disponibilidad media anual de las aguas superficiales en las cuencas hidrológicas Ixtapa, Pitillal, Cuale, San Blas, Huicicila y Tecomala, las cuales forman parte de la Región Hidrológica número 13 Río Huicicila. Asimismo, se consultó el Acuerdo (publicado el 28 de agosto de 2009) por el que se da a conocer la ubicación geográfica de 371 acuíferos del territorio nacional, se actualiza la disponibilidad media anual de agua subterránea de 282 acuíferos, y se modifica, para su mejor precisión, la descripción geográfica de 202 acuíferos

Para la disponibilidad de agua subterránea, según el Diario Oficial de la Federación -mediante Acuerdo por el que se da a conocer el resultado de los estudios técnicos de aguas nacionales subterráneas del acuífero Valle Santiago-San Blas, clave 1803, en el Estado de Nayarit, Región Hidrológico-Administrativa Lerma-Santiago-Pacífico y publicado el 22 de abril de 2016-, se tomó la región 
hidrológica administrativa 1803 valle Santiago-San Blas para la que se reportó 31.525.653 $\mathrm{m}^{3}$ de agua por año. Se hizo una corrección mediante una distribución per cápita de las personas que se abastecen de la cuenca (468.402) y considerando la población de la zona costera de San Blas.

Para el cálculo del agua superficial, se consultó el Acuerdo en el que se reporta para la cuenca de San Blas una disponibilidad de $362.370 .000 \mathrm{~m}^{3}$ por año y se distribuyó entre el número de personas beneficiadas y se ajustó a la población de la zona costera de San Blas.

Se realizó el ajuste del WTA (según el método de Pfister) de acuerdo con la variación de la precipitación anual (ecuación (3)).

$$
W T A^{*}=\sqrt{V F} \cdot W T A
$$

\section{Donde:}

$W T A^{*}=$ grado de presión hídrica ajustado a la variación de la precipitación anual.

$V F=$ desviación estándar de la distribución pluvial (el valor estándar para México es 3,24).

$W T A=$ grado de presión hídrica.

Por otro lado, debido a que la presión hídrica no es lineal respecto a WTA*, en la metodología de Pfister se realiza un ajuste al valor de WSI a una función logarítmica que proporciona valores continuos entre 0,01 y 1, según la ecuación (4).

$$
W S I=\frac{1}{1+e^{-6.4 W T A^{*}}\left(\frac{1}{0.10}-1\right)}
$$

Una vez obtenido el índice de estrés hídrico (WSI), se calculó la huella de agua (HA), a través de multiplicar el valor del índice de estrés hídrico, por el volumen del agua distribuido, por el porcentaje de cobertura de agua potable. El porcentaje de agua potable $(93,71 \%)$ para la zona costera de San Blas se estimó con base al censo del INEGI 2010. Para el volumen de distribución se consideró el de la Lerma-Santiago-Pacífico que es de $98,9 \mathrm{~m}^{3} /$ hab/año.

\section{RESULTADOS}

Los resultados de este trabajo indican que, en la zona costera de San Blas, el recurso hídrico no se encuentra en estrés, pues existe una buena disponibilidad. La huella de agua por el método de escasez se puede considerar como un indicador de la proporción de las extracciones anuales y la disponibilidad de agua; permite realizar comparaciones de la presión a la que se encuentra sometido el recurso hídrico entre zonas, regiones y países. Además, puede contribuir a la planeación de la distribución y manejo del agua en el uso agrícola y urbano, en regiones como la del presente estudio, donde la superficie de uso agrícola supera el $35 \%$. En este sentido, la huella de agua en la zona costera de San Blas, que es el volumen total de agua utilizada por los habitantes, es de 3,6904 m/ hab/año. Este valor queda por debajo de la huella de agua nacional que es de $4,161 \mathrm{~m}^{3} / \mathrm{hab} / \mathrm{año}$ (Farell, Turpin y Suppen, 2013). La Región hidrológica administrativa (RHA) con mayor Huella de agua es la II Noroeste con 226,485 m³/hab/año (Farell, Turpin y Suppen, 2013).

El grado de presión hídrica (WTA) para la zona fue de $12,26 \%$, que se considera moderado (Vorosmarty et al., 2000). De aquí, se considera que esta zona no presenta estrés hídrico, ya que la extracción de agua no supera su disponibilidad. Este WTA es inferior al promedio nacional reportado por Farell, Turpin y Suppen (2013) que es de 17,4\%. La región hidrológica administrativa (RHA) de México que tiene mayor WTA es la XIII Aguas del Valle de México con 132,3\%, y la menor es la XI Frontera Sur con 1,4 \% de WTA. La RHA Lerma-Santiago-Pacífico, en la que se localiza la zona de estudio, tiene un WTA de 41,5 \% (Farell, Turpin y Suppen, 2013). No siempre un sitio con grado de presión fuerte (WTA) tiene una huella alta. Por otro lado, el índice de estrés hídrico (WSI) para la zona fue de 0,0398. Este valor resulta inferior al total del país, que es de 0,3125 (Farell, Turpin y Suppen, 2013). 


\section{CONCLUSIONES}

La estimación de la huella de agua presentada en este trabajo considera la relación entre la extracción de agua dulce para usos humanos y su disponibilidad total en cierta región (WSI), el volumen distribuido y el porcentaje de cobertura de agua potable de la zona en estudio. Se recomienda que, en este tipo de estimaciones, en caso de tener la información, se incluyan la medición de otras variables que afectan el agua como la acidificación, eutrofización, ecotoxicidad y calor, a fin de proporcionar una imagen completa de los impactos relacionados con el agua.

En trabajos futuros, para mostrar una perspectiva integral sobre la crisis de agua, sería conveniente incluir en este tipo de análisis variables económicas, sociales y ambientales que ofrezcan una conexión entre políticas, mercado y usos del agua, con el propósito de regular la extracción, uso y disposición del recurso agua, para evitar que la demanda de agua dulce exceda el abastecimiento.

\section{REFERENCIAS}

Alcamo, J., Henrichs, T. y Rosch, T. (2000). World Water in 2025: Global Modeling and Scenario Analysis for the World Comission on Water for the $21^{\text {st }}$ century. Kassel, Alemania: University of Kassel. Recuperado de https://wedocs.unep.org/ bitstream/handle/20.500.11822/19071/World_ water_in_2025_global_modeling_scenarios. pdf? sequence $=1$ \&isAllowed $=y$

Allan, J. A. (1998). Virtual water: a strategic resource. Ground water, 36(4), 545-547. https://doi.org/10.1111/j.1745-6584.1998.tb02825.x

Boulay, A., Bare, J., Benini, L., Berger, M., Bulle, C., Klemmayer, I., ..., Pfister, S. (2015). The WULCA consensus characterization model for water scarcity footprints: assessing impacts of water consumption based on available water remaining (AWARE). The International Journal of Life Cycle Assessment, 23(2), 368-378. https://doi.org/10.1007/ s11367-017-1333-8
Comisión Nacional del Agua (Conagua) (2017). Estadísticas del agua en México. Edición 2017. Secretaría de Medio Ambiente y Recursos Naturales. Recuperado de www.conagua.gob.mx

Comisión Nacional del Agua (Conagua) (2017). Estadísticas del agua en México. Edición 2017. Secretaría de Medio Ambiente y Recursos Naturales, p. 77. Recuperado de http://sina.conagua.gob.mx/publicaciones/EAM_2017.pdf

Damkjaer, S. y Taylor, R. (2017). The measurement of water scarcity: Defining a meaningful indicator. Ambio, 46(5), 513-531. https://doi.org/10.1007/ s13280-017-0912-Z

De Quevedo, F., Asprilla, Y. y González, M. (2017). Entropías de la movilidad urbana en el espacio metropolitano de Guadalajara: transporte privado y calidad del aire. Tecnura, 21(53), 138-149. https:// doi.org/10.14483/22487638.10725

Falkenmark, M. (1986). Fresh water: Time for a modified approach. Ambio, 4(15), 192200. Recuperado de https://www.jstor.org/ stable/4313251? seq=1\#page_scan_tab_contents

Falkenmark, M. (1989). The massive water scarcity threatening Africa-why isn't it being addressed. Ambio, 2(18), 112-118. Recuperado de https://www.jstor.org/stable/pdf/4313541. pdf?seq=1\#page_scan_tab_contents

Farell, C., Turpin, C. y Suppen, N. (2013). Huella de agua de uso público-urbano en México. Revista Internacional de Estadística y Geografía, 1(4), 5871. Recuperado de https://www.inegi.org.mx/rde/ RDE_08/Doctos/RDE_08_Art4.pdf

Food and Agriculture Organization of the United Nations (FAO) (2018). The Global Framework on Water Scarcity in Agriculture. Roma. Recuperado de http://www.fao.org/land-water/overview/wasag/en/

Frischknecht, R., Steiner, R., Braunschweig, A., Egli, N. y Hildesheimer, G. (2006). Swiss ecological scarcity method: the new version 2006. Berna, Suiza. Recuperado de https://www.researchgate.net/publication/237790160_Swiss_Ecological_Scarcity_ Method_The_New_Version_2006

Gleick, P.H. (1996). Basic Water Requirements for Human Activities: Meeting Basic Needs. 
Water International (IWRA), 21(2), 83-92. https:// doi.org/10.1080/02508069608686494

González, N., Carvajal, Y. y Loaiza, W. (2016). Análisis de sequías meteorológicas para la cuenca del río Dagua, Valle del Cauca, Colombia. Tecnura, 20(48), 101-113. DOI: 10.14483/udistrital.jour. tecnura.2016.2.a07

González, G., Retamoza, G., Álborez, H. y Guerrero, A. (2016). Gestión integral de cuencas hidrográficas: una alternativa a la sustentabilidad de los recursos hídricos en México. Lacandonia, 1(10), 91-98. Recuperado de https://cuid.unicach.mx/revistas/index.php/lacandonia/article/down load/398/364/

Hanafiah, M., Xenopoulos, A., Pfister, S., Leuven, R. y Huijbregts, M. (2011). Characterization factors for water consumption and greenhouse gas emissions based on freshwater fish species extinction. Environment Science Technology, 45(12), 5272-5278. https://doi.org/10.1021/es1039634

Hoekstra, A.Y., Chapagain, K., Aldaya, M. y Mekonnen, M. (2011). The water footprint assessment manual: setting the global standard. Londres/Washington: Earhscan Ltd. Recuperado de https://waterfootprint. org/media/downloads/TheWaterFootprintAssessmentManual_2.pdf

Hoekstra, A.Y. (ed.) (2003). Virtual water trade: Proceedings of the International Expert Meeting on Virtual Water Trade. Delft, Países Bajos: Unesco-IHE. Recuperado de https://waterfootprint.org/media/ downloads/Report12.pdf

Hoekstra, A.Y. y Chapagain, A.K. (2004). Water footprints of nations. Vol. 1 Main report. Delft, Países Bajos: Unesco-IHE. Recuperado de https://waterfootprint.org/media/downloads/Report16Vol1.pdf

Instituto Nacional de Geografía (INEGI) (2010). Principales resultados por localidad (ITER). Recuperado de: https://www.inegi.org.mx/programas/ ccpv/2010/default.html\#Datos_abiertos

Martínez, A., Chargoy, J., Puerto, M., Suppen, N., Rojas, D., ..., Villarraga J. (2016). Huella de agua (ISO 14046) en América Latina. Aanálisis y recomendaciones para una coherencia regional. Centro de Análisis de Ciclo de Vida y Diseño Sustentable CADIS, Embajada de Suiza en Colombia, Agencia
Suiza para la Cooperación y el Desarrollo COSUDE. Recuperado de https://www.shareweb.ch/ site/Suiz-Agua-Colombia/Documents/Huella\%20 Agua\%20ISO\%2014046\%20America\%20Latina. pdf

Motoshita, M., Itsubo, N. e Inaba, A. (2010). Development of impact factors on damage to health by infectious diseases caused by domestic water scarcity. The International Journal of Life Cycle Assessment, 16(1), 65-73. https://doi.org/10.1007/ s11367-010-0236-8

Ohlsson, L. (2000). Water Conflicts and Social Resource Scarcity. Phys. Chem. Earth, 3(25), 213-220. https:// doi.org/10.1016/S1464-1909(00)00006-X

Organización de las Naciones Unidas para la Alimentación y la Agricultura (FAO) (2018). Afrontar la escasez de agua. Un marco de acción para la agricultura y la seguridad alimentaria. Informe sobre temas hidricos 38. Roma, Italia. Recuperado de http://www. fao.org/3/a-i3015s.pdf

Pfister, S., Koehler, A. y Hellweg, S. (2009). Assessing the environmental impact of freshwater consumption in LCA. Environ. Sci. Technol., 43(11), 40984104. https://doi.org/10.1021/es802423e

Rees, W.E. (1992). Ecological footprints and appropriated carrying capacity: what urban economics leaves out. Environment and Urbanization, 4(2), 121-130. https://doi.org/10.1177/095624789200400212

Rees, W.E. (1996). Revisiting carrying capacity: area-based indicators of sustainability. Population and Environment, 17(3), 195-215. https://doi.org/10.1007/ BF02208489

Ress, W.E.yWackernagel, M. (1992). Ecological footprints and appropriated carrying capacity: Measuring the natural capital requirements of the human economy. Environment and Urbanization, 4(2), 121-130. https://doi.org/10.1177/095624789200400212

Ress, W.E. y Wackernagel, M. (1996). Urban ecological footprints: why cities cannot be sustainable and why they are a key to sustainability. Environmental Impact Assess, (16), 223-248. https://doi. org/10.1016/S0195-9255(96)00022-4

Seckler, D., Molden, D. y Barker, R. (1999). Water scarcity in the twenty-first century. International Journal 
of Water Resources Development 15(1-2):29-42. https://doi.org/10.1080/07900629948916

Suarez, F., García, A. y Vaca, L. (2012). Identificación y evaluación de la contaminación del agua por curtiembres en el municipio de Villapinzón. Tecnura, O(16), 185-194. Recuperado de https://www.redalyc.org/pdf/2570/ 257025147015.pdf

Sullivan, C. (2002). Calculating a Water Poverty Index. World Development, 30(7), 1195-1210. https://doi. org/10.1016/S0305-750X(02)00035-9

Taylor, R. (2009). Rethinking water scarcity: The role of storage. Eos, Transactions American Geophysical Union, 28(90), 237-238. https://doi. org/10.1029/2009EO280001

Van Zelm, R., Schipper, A., Rombouts, M., Snepvangers, J. y Huijbregts, M. (2010). Implementing groundwater Extraction in Life Cycle Impact Assessment: Characterization Factors Based on Plant Species Richness for the Netherlands. Environmental Science \& Technology, 45(2), 629-635. https:// doi.org/10.1021/es102383v

Vorosmarty, J., Green, P., Salisbury, J. y Lammers, R., B. (2000). Global water resources: Vulnerability from climate change and population growth. Science, 289(5477), 284-288. https://doi.org/10.1126/ science.289.5477.284

World Economic Forum (2016). The Global Risks Report 2016. Edition 11th. Ginebra, Suiza: The Global Competitiveness and Risk Team. Recuperado de http://www3.weforum.org/docs/GRR/WEF_ GRR16.pdf 